\title{
O086: Antibiotic use and vancomycin-resistant enterococcus (VRE) carriage during a large outbreak in a dutch hospital
}

\author{
J van Balveren ${ }^{1}$, LB van der Velden ${ }^{1 *}$, HW Fleuren², MH Nabuurs-Franssen ${ }^{1}$, A Voss ${ }^{1,3}$, T Sprong ${ }^{1,4}$ \\ From 2nd International Conference on Prevention and Infection Control (ICPIC 2013) \\ Geneva, Switzerland. 25-28 June 2013
}

\section{Introduction}

In 2012 a large number of Dutch hospitals experienced outbreaks of vancomycin-resistant enterococcus (VRE), including our (560 beds) secondary care hospital.

\section{Objectives}

To examine whether (previous) antibiotic use was associated with VRE-carriage.

\section{Methods}

We studied all patients who were found positive for VRE carriage (rectal swab) by PCR and or culture during admission from an outbreak in a large (560 beds) secondary care hospital in the Netherlands, in the period May to August 2012. From these 120 patients antibiotic use was retrieved from the database of our electronic prescription system.

\section{Results}

From the 120 patients identified as VRE carrier, 112 $(93,3 \%)$ had used one or more antibiotics in the 3 months before VRE-positivity. 78 (69.6\%) patients had used ciprofloxacin and $52(46.6 \%)$ a third-generation cephalosporin (ceftriaxone or cefotaxime). Interestingly, only 1 patient $(0.9 \%)$ had used vancomycin in the 3 months before positivity. Median duration of admission and time to VRE-positivity were significantly longer in the patients who had received prior antibiotic therapy (14 days) compared to the patients who did not receive antibiotic therapy (4 days).

\section{Conclusion}

VRE-carriage is almost exclusively seen in patients who have previously received antimicrobial therapy. The short time to positivity in the patients who did not receive previous antibiotic therapy may suggest that they acquired VRE in the community or during an admission $>3$ months before VRE positivity. In this outbreak, vancomycin use was not related to VRE carriage. A retrospective case control study will be performed on these patients to identify which antibiotics predispose to VRE-carriage.

\section{Disclosure of interest}

None declared.

\section{Author details}

${ }^{1}$ Medical Microbiology and Infectious Diseases, Nijmegen, The Netherlands. ${ }^{2}$ Clinical Pharmacology, Canisius Wilhelmina Hospital Nijmegen, Nijmegen, The Netherlands. ${ }^{3}$ Medical Microbiology, University Hospital Nijmegen Medical Center, Nijmegen, The Netherlands. ${ }^{4}$ Internal Medicine, Canisius Wilhelmina Hospital Nijmegen, Nijmegen,The Netherlands.

Published: 20 June 2013

\section{doi:10.1186/2047-2994-2-S1-086}

Cite this article as: van Balveren et al:: O086: Antibiotic use and vancomycin-resistant enterococcus (VRE) carriage during a large outbreak in a dutch hospital. Antimicrobial Resistance and Infection Control 2013 2(Suppl 1):086 